\title{
First detection of dust clouds around R CrB variable stars ${ }^{\star}$
}

\author{
P. de Laverny and D. Mékarnia
}

\begin{abstract}
Observatoire de la Côte d'Azur, Dpt Cassiopée, CNRS - UMR 6202, BP 4229, 06304 Nice Cedex 4, France e-mail: [laverny;mekarnia]@obs-nice.fr
\end{abstract}

Received 6 September 2004 / Accepted 23 October 2004

\begin{abstract}
From VLT/NACO diffraction-limited images of RY Sgr, we report the first direct detection of heterogeneities in the circumstellar envelope of a $\mathrm{R}$ Coronae Borealis variable star. Several bright and very large dust clouds are seen in various directions at several hundred stellar radii from RY Sgr, revealing high activity for the ejection of stellar material by $\mathrm{R}$ CrB variables. These observations do support the current interpretation that optically thick dust clouds are formed around the surface of this type of variable stars and, when passing between the star and the observer, produce the huge and sudden declines characterizing these objects in visible light. This is the first direct confirmation of a scenario proposed about 70 years ago.
\end{abstract}

Key words. stars: AGB and post-AGB - stars: variables: general - stars: individual: RY Sgr - stars: mass-loss stars: circumstellar matter - techniques: high angular resolution

\section{Introduction}

"Le R Coridini (protebbero), per qualche motivo, cingersi (....) di gas oscuranti emessi a tratti da queste stelle stesse". In this way, Loreta (1934) was the first to propose that R Coronae Borealis type variables ( $\mathrm{R} \mathrm{CrB}$, hereafter) might eject absorbing material responsible of the huge brightness declines (or fadings) characterizing these stars in visible light. Then, O'Keefe (1939) showed that this ejected material could condense at small distances from the central star and form obscuring clouds rich in carboneous compounds. These clouds would then gradually dissipate as they are driven away by radiation pressure.

For more than 70 years, this scenario for the interpretation of the light variations of $\mathrm{R} \mathrm{CrB}$ variable stars has been widely accepted: the fadings are believed to be caused by obscurations of the stellar surface by newly formed dusty and optically thick clouds. These stars indeed exhibit drastic and erratic variabilities. Their visual lightcurve is characterized by unpredicted decreases of up to $8 \mathrm{mag}$, in a time-scale of weeks. The return to normal light is much slower and can last up to several months (see Clayton 1996). There are several observational evidences in favor of this scenario (see Clayton 1996, for more details). Among them, the fact that (i) the dust consists primarily as amorphous carbon particles; (ii) mass-loss rates are as large as $10^{-6} M_{\odot} /$ year and episodic, with timescales of a few months; (iii) mass-loss is driven by fast winds (Clayton et al. 2003); (iv) the dust may form only over a small solid angle of the stellar surface (perhaps the cool regions above large convective cells, as first proposed by Wdowiak 1975)

\footnotetext{
* Based on observations collected with the VLT/UT4 Yepun telescope (Paranal Observatory, ESO, Chile) using the NACO instrument (program ID 71.D-0543A).
}

or is ejected in some specific directions (Clayton et al. 1997); (v) polarimetric observations may reveal the presence of permanent clumpy non-spherical dust shells (Clayton et al. 1997; Yudin et al. 2003); (vi) larger polarizations are seen during declines revealing more scattering particles between the star and the observer (see references in Clayton 1996); (vii) characteristic time-scales of the light variations are compatible with the formation of dust clouds close to the stellar photosphere and their dilution in the outer regions (Hartmann \& Apruzese 1976; Zubko 1997).

However, in spite of the above evidences, the dusty environment close to the star remains almost unknown: no direct detection of the suspected heterogeneities in the dust distribution in the circumstellar envelope of a $\mathrm{R} \mathrm{CrB}$ variable have up to now been achieved. Observations of these inner layers in R CrB were reported by Ohnaka et al. (2003, and previous references) but no significant deviation from circular geometry was detected (perhaps because of the rather small dynamical range of their observations, inherent to the technique adopted). In this letter, we present the first direct detection of the presence of dusty clouds around RY Sgr, the brightest $\mathrm{R} C r B$ variable in the southern hemisphere.

\section{Observations}

Observations of RY Sgr were performed in service mode in May and September 2003 using the adaptive optics system NACO at the Nasmyth-B focus of the ESO/VLT fourth 8-m telescope unit Yepun located at Cerro Paranal, Chile. $\mathrm{NACO}$, which is an association of the adaptive optics system NAOS (Rousset et al. 2002) and the spectro-imager CONICA (Lenzen et al. 2002), provides diffraction-limited 
Table 1. Observations log.

\begin{tabular}{lccc}
\hline \hline $\begin{array}{l}\text { Date } \\
(\mathrm{UT})\end{array}$ & $\begin{array}{c}\lambda_{\mathrm{c}} \\
(\mu \mathrm{m})\end{array}$ & $\begin{array}{c}\text { Exp. time } \\
(\text { on-source in min) }\end{array}$ & $\begin{array}{c}\text { Seeing } \\
\left({ }^{\prime \prime}\right)\end{array}$ \\
\hline 2003 May 17 & 1.04 & 80 & $\sim 0.8$ \\
2003 May 24 & 2.17 & 33 & $\sim 0.5$ \\
2003 September 17 & 4.05 & 3 & $\sim 0.9$ \\
\hline
\end{tabular}

images in the near-infrared $(1-5 \mu \mathrm{m})$ spectral range (see http: //www . eso.org/instruments/naco/).

RY Sgr was observed using three narrow-band filters: NB 1.04 (centered at $\left.\lambda_{\mathrm{c}}=1.04 \pm 0.015 \mu \mathrm{m}\right), \mathrm{NB} 2.17\left(\lambda_{\mathrm{c}}=\right.$ $2.166 \pm 0.023 \mu \mathrm{m})$ and NB $4.05\left(\lambda_{\mathrm{c}}=4.051 \pm 0.02 \mu \mathrm{m}\right)$. The pixel scale on CONICA was respectively 13.25 mas in the NB 1.04 and NB 2.17 filters and 27.03 mas in the NB 4.05 filter, adapted to the observing wavelengths. The seeing conditions were variable, ranging from $\sim 0.5^{\prime \prime}$ during observations at $2.17 \mu \mathrm{m}$ to $\sim 0.9^{\prime \prime}$ during observations at $4.05 \mu \mathrm{m}$. The AutoJitter mode was used, i.e. that at each exposure, the telescope was moved according to a random pattern in a $6^{\prime \prime} \times 6^{\prime \prime}$ box. The sky is then estimated from all the observations. To allow further resolution improvement through deconvolution, a PSF reference star (HD 178199) was observed immediately after each RY Sgr observation using the same configuration of the adaptive optics system. The air-masses of RY Sgr and of the PSF reference star were 1.0 and 1.1 respectively and NAOS was servoed on RY Sgr itself. The FWHM of the PSF, estimated from the reference star in the different narrow-band filters, was $0.058^{\prime \prime}, 0.068^{\prime \prime}$ and $0.116^{\prime \prime}$ at $1.04 \mu \mathrm{m}, 2.17 \mu \mathrm{m}$ and $4.05 \mu \mathrm{m}$ respectively. Calibration files (flat fields and dark exposures) were acquired, following the ESO/VLT standard calibration plan. The total on-source integration time in the three filters were $80 \mathrm{mn}, 33 \mathrm{mn}$ and $3 \mathrm{mn}$ respectively. The dynamic range of the final images goes from 3300 at $2.17 \mu \mathrm{m}, 12500$ at $1.04 \mu \mathrm{m}$ to 54000 at $4.05 \mu \mathrm{m}$. Table 1 summarizes the observational conditions.

Standard reduction procedures were applied using selfdeveloped routines. The raw images were sky subtracted, then divided by the flat-field and corrected from hot pixels. In each filter, the images were cross-correlated and aligned by subpixel shifting, and then combined to produce the final images, eliminating discrepant points like cosmic rays. Finally, the images were deconvolved with the PSF reference star. We used the Richardson-Lucy algorithm (Richardson 1972; Lucy 1974), which appears to be more suitable for data with a high dynamic range, such as ours. Constancy of prominent features present in deconvolved images showed that the PSF selection and the number of iterations (10-20 iterations, typically) for the deconvolution was performed carefully and conservatively.

\section{Images of the circumstellar envelope of RY Sgr}

The diffraction-limited images of RY Sgr at $1.04 \mu \mathrm{m}, 2.17 \mu \mathrm{m}$ and $4.05 \mu \mathrm{m}$ are presented in Figs. 1 and 2. The images are displayed with a log-scale for the brightness, so that details of the morphology at all flux levels can be seen. In the $1.04 \mu \mathrm{m}$ image (Fig. 1) collected at the same epoch as the $2.17 \mu \mathrm{m}$ one

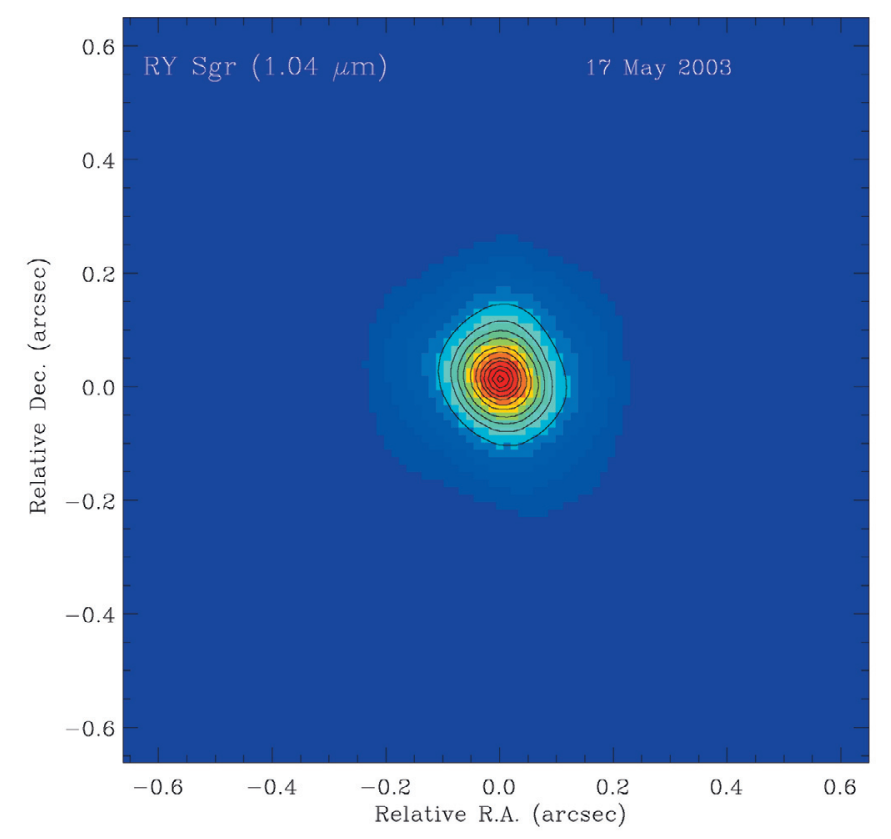

Fig. 1. NACO image of RY Sgr at $1.04 \mu \mathrm{m}$. Contour levels are superimposed on the brightness-color log-scale of the image. They are 90 , $70,50,30,20,10,5,2,1,0.5$ and $0.2 \%$ of the peak surface brightness. North is up, and east is to the left. The spatial resolution estimated from the PSF reference star is 58 mas.

(Fig. 2, left), RY Sgr is not resolved and no structures are seen in its envelope. On the other hand, RY Sgr reveals strong departures from a point-like source at longer wavelengths since several structures are seen in the $K(2.17 \mu \mathrm{m})$ and $L(4.05 \mu \mathrm{m})$ images (Fig. 2). In this section, we describe only the brightest detected heterogeneities, i.e. those appearing brighter than $0.2 \%$ of the peak level and thus well above the noise level defined by the dynamics of the images.

In the $K$-band, two elongations are clearly seen towards the NW and SW directions (at PA $\sim 330^{\circ}$ and $\mathrm{PA} \sim 195^{\circ}$ respectively). These bright structures are located at about $0.1^{\prime \prime}$ from the central star and they appear to have a typical size similar to the one of the central star itself.

At longer wavelengths $(4.05 \mu \mathrm{m})$, the departure from a point-like symmetry is even more evident. The most prominent structures are two clouds as bright as $2 \%$ of the stellar peak. They are found at about $0.2^{\prime \prime}$ from the center of the image (i.e. twice further than in the $K$-image, revealing that different clouds are seen in the $L$-band) at $\mathrm{PA} \sim 20^{\circ}$ and $\mathrm{PA} \sim 260^{\circ}$. Their size is of the order of $0.2^{\prime \prime}$.

Finally, fainter structures are seen in both images of Fig. 2. These structures could be artifacts in $K$-band but they are about 10 times brighter than the noise level in the $L$-band image. Therefore, RY Sgr might be surrounded at a given epoch by several clouds (about ten or so) located at distances up to $0.5^{\prime \prime}$ from its center. Nevertheless, this more complex configuration should be confirmed by new independent observations. 

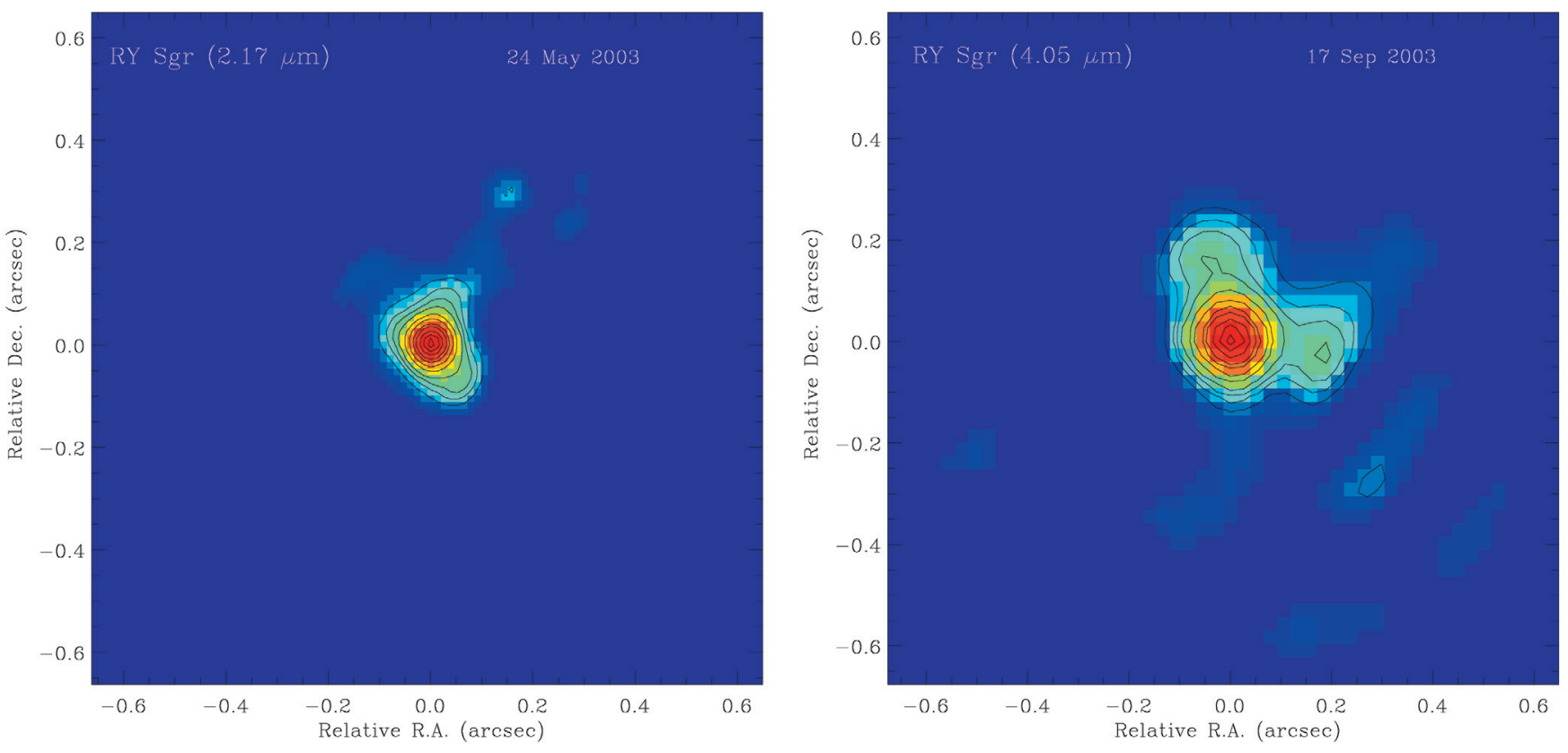

Fig. 2. NACO images of RY Sgr at $2.17 \mu \mathrm{m}$ (left) and $4.05 \mu \mathrm{m}$ (right, collected 4 months later). Same contour levels and orientation as Fig. 1. The pixel scale at $4.05 \mu \mathrm{m}$ is twice larger due to NACO characteristics. The spatial resolution estimated from the PSF reference star is 68 mas and 116 mas at $2.17 \mu \mathrm{m}$ and $4.05 \mu \mathrm{m}$, respectively. Note that the structures seen in both images result from different ejection events (see text).

\section{Discussion}

These images do reveal undoubtedly that large clouds are present in the vicinity of $\mathrm{R} \mathrm{CrB}$ variables. They are seen in different directions and located at different distances from the central star. It has to be pointed out that the clouds seen in the $K$ and $L$-images result from different ejection events. Indeed, an extremely large velocity (more than one order of magnitude above the typical escape velocity observed around $\mathrm{R} C \mathrm{CrB}$ variables) would be required to explain such a cloud motion ( $0.1^{\prime \prime}$ in 4 months), at the estimated distance of RY Sgr ( $\sim 2 \mathrm{kpc}$, see below). We also note that the clouds appearing at $\sim 0.2^{\prime \prime}$ from the center of the field in the $L$-band image are not seen in $K$-band because, despite the better spatial resolution, the dynamic range in $K$ may not be high enough to detect these rather cold and therefore too faint clouds. On another hand, the structures seen in May 2003 in $K$-band are not detected in September in $L$-band. A possible explanation is that the angular resolution of the $L$ image $\left(0.116^{\prime \prime}\right)$ is not high enough to resolve these features that are present at about $0.1^{\prime \prime}$ from the center of the field. In any cases, it is very likely that these clouds are composed of dust particles since they are not detected at $1.04 \mu \mathrm{m}$ while they clearly appear at the same epoch in $K$-band where their emission is high enough to be detected.

Therefore, these observations do confirm, for the first time, the scenario proposed several decades ago by Loreta (1934) and O'Keefe (1939) and now widely accepted. When a very optically thick dust cloud is ejected towards the observer, a huge brightness decline, characteristic of $\mathrm{R} \mathrm{CrB}$ variable stars, is observed in visible light. On the contrary, almost no variations are seen at longer wavelengths where this cloud is optically thin.

The rather large number of clouds detected around RY Sgr also reveals a high activity for the $\mathrm{R} C r B$ variable stars regarding the ejection of stellar material. Such large departures from spherical symmetry around RY Sgr could explain why brightness declines of $\mathrm{R} \mathrm{CrB}$ variables are not so rare (a few every 10 years, typically): a larger number of ejected clouds (if the ejection is isotropic) leads to a larger probability that one of them lies on the line of sight. R CrB stars with the most frequent brightness declines would therefore be the stars ejecting material at the larger rate. For instance, RY Sgr exhibited about 10 declines over the last 50 years (from AAVSO lightcurve). That is a frequency about half that of the star $\mathrm{R} \mathrm{CrB}$, which should be surrounded by a higher number of dust clouds and might therefore eject material at a higher rate.

In addition, not only the number of ejected clouds is large but these clouds might be very dense and optically thick close to the stellar surface. It has indeed to be noted that the mean density of the circumstellar layers, where they are detected in the NACO images, is a factor of the order of $10^{6}$ lower (assuming a density law varying as $r^{-2}$ in the envelope) with respect to the regions very close to the star where they are formed. If we assume that the density in the clouds has decreased by the same amount, it results that an impressive quantity of material is suddenly ejected from the stellar surface and eventually form the dust clouds surrounding these stars.

Furthermore, the dusty clouds are detected rather far from RY Sgr itself, shedding new light on their dilution into the interstellar medium. RY Sgr is a rather hot $\mathrm{R} \mathrm{CrB}$ variables $\left(T_{\text {eff }}=7000-7500 \mathrm{~K}\right.$, following Asplund et al. 2000). It could thus belong to the class of the brightest $\mathrm{R} \mathrm{CrB}$ with $M_{V}=-5$ as revealed by SMC \& LMC R CrB variables (Alcock et al. 2001; Tisserand et al. 2004, assuming that galactic R CrB stars have similar properties). Adopting a bolometric correction $\mathrm{BC}=-0.15$ for a $\mathrm{G} 0$ supergiant, the radius of $\mathrm{RY} \mathrm{Sgr}$ 
is approximately $60 R_{\odot}$. From the adopted absolute magnitude and the AAVSO photometry ( $m_{V} \sim 6.4$ at maximum light), we estimate that RY Sgr lies at about $1.9 \mathrm{kpc}$, yielding an angular radius 0.15 mas for this star. The dust clouds shown in Fig. 2, placed at about $0.1-0.2^{\prime \prime}$, are therefore located between $\sim 700-1400 R_{*}$ and their typical radius is also close to a few hundred stellar radii. That offers new constraints on the dilution of these clouds in the outer circumstellar envelopes of $\mathrm{R} \mathrm{CrB}$ variables, since they still have an important size far from the region where they were formed. The NACO images indeed reveal that the light recovery of $\mathrm{R} \mathrm{CrB}$ stars in the optical would not be caused by the evaporation of the clouds close to the stellar surface since they are still seen far from the central object. These clouds are indeed rather steady because they have been ejected a few years ago (about 5-10 years, assuming a typical escape velocity of at least $200 \mathrm{~km} \mathrm{~s}^{-1}$, Clayton et al. 2003). We also note that, if the most distant structures seen at about $0.5^{\prime \prime}$ (or more than $3000 R_{*}$ ) are confirmed, they might have been ejected a few decades ago. This reveals that dust clouds around $\mathrm{R} \mathrm{CrB}$ variables move away from the star leading, if present on the line of sight, to less obscuration of the surface. Therefore, the return to maximum light might not be caused by the evaporation of the clouds very close to the star.

Finally, regarding the location of the formation of the dust clouds, we cannot discriminate from the collected data with insufficient spatial resolution between the two commonly adopted scenarios: either the dust is formed very close to the stellar surface $\left(\sim 2 R_{\star}\right)$ or it is formed in regions more distant than $\sim 20 R_{\star}$ (see Clayton 1996).

\section{Summary and conclusion}

We have presented the first detection of heterogeneities in the circumstellar envelope of a $\mathrm{R}$ Coronae Borealis variable star owing to high spatial resolution images collected with NACO.

Several large dust clouds are found in different directions at several hundred stellar radii from RY Sgr. These observations do support the current interpretation that the huge and sudden declines which characterize these objects in optical light are caused by the formation of dust clouds along the line of sight. However, the present data are not sufficiently spatially resolved to verify whether the dust is formed close to the stellar surface or in more distant regions. Future observations with higher spatial resolutions (ideally a factor 10 or more), also with very high dynamics, should help to better understand the circumstellar environment and the evolution of these variable stars.

Acknowledgements. We thank A. P. P. Recio-Blanco for her careful reading of the manuscript and her Anglo-Italian languages expertise. G. Niccolini is acknowledged for comments and discussions on this work. The referee (G. C. Clayton) and M. Asplund are thanked for their valuable suggestions. We are also greatful to the variable star observations from the AAVSO International Database, contributed by observers worldwide and used in this research.

\section{References}

Alcock, C., Allsman, R. A., Alves, D. R., et al. 2001, ApJ, 554, 298

Asplund, M., Gustafsson, B., Lambert, D. L., \& Rao, N. K. 2000, A\&A, 353, 287

Clayton, G. C. 1996, PASP, 108, 225

Clayton, G. C., Bjorkman, K. S., Nordsieck, K. H., et al. 1997, ApJ, 476,870

Clayton, G. C., Geballe, T. R., \& Luciana, L. 2003, ApJ, 595, 412

Hartmann, L., \& Apruzese, J. P. 1976, ApJ, 203, 610

Lenzen, R., Hartung, M., Brandner, W., et al. 2002, SPIE, 4841

Loreta, E. 1934, Astron. Nach., 254, 151

Lucy, L. B. 1974, AJ, 79, 745

Ohnaka, K., Beckmann, U., Berger, J.-P., et al. 2003, A\&A, 408, 553

O'Keefe, J. A. 1939, ApJ, 90, 294

Richardson, W. H. 1972, JOSA, 62, 55

Rousset, G., Lacombe, F., Puget, P., et al. 2002, SPIE, 4007

Tisserand, P., Marquette, J. B., Beaulieu, J. P., et al. 2004, A\&A, 424, 245

Wdowiak, T. J. 1975, ApJ, 198, L139

Yudin, R. V., Evans, A., Barrett, P., et al. 2003, A\&A, 412, 405

Zubko, V. G. 1997, MNRAS, 289, 305 